\title{
THE STATUS OF THE DARESBURY ENERGY RECOVERY LINAC PROTOTYPE
}

\author{
S.L.Smith, N.Bliss, A.R.Goulden, D.J.Holder, P.A.McIntosh, G.Priebe, STFC Daresbury \\ Laboratory, Warrington, UK.
}

\section{Abstract}

As part of the UK's R\&D programme to develop an advanced energy recovery linac-based light source (4GLS) a $35 \mathrm{MeV}$ technology demonstrator called the Energy Recovery Linac Prototype (ERLP) has been constructed. It is based on a combination of a DC photocathode electron gun, a superconducting injector linac and main linac operating in energy recovery mode, driving an IR-FEL. The priorities for this machine are to gain experience of operating a photoinjector gun and superconducting linacs; to produce and maintain highbrightness electron beams; to achieve energy recovery from an FEL-disrupted beam and to study challenging synchronisation issues. The current status of this project is presented, including construction and commissioning progress and plans for the future exploitation of this scientific and technical R\&D facility.

\section{INTRODUCTION}

Since the status of this project was last reported [1] there have been a number of significant milestones achieved:

- First electrons obtained from the gun;

- Operation of the cryogenic system to cool both superconducting linac modules to $2 \mathrm{~K}$;

- Demonstration of RF-photoinjector drive laser synchronisation;

- Initial high power RF tests of the linac module;

- Commissioning of a terawatt laser to drive a Compton backscattering x-ray source and electrooptic longitudinal beam diagnostics.

In addition, this progress has been made in parallel with an extensive construction programme to complete the installation of the whole machine (barring a few diagnostic devices currently being used with the gun) in preparation for commissioning of the completed machine later this year. The layout of the ERLP is illustrated in Fig. 1, while the key parameters are listed in Table 1.

Table 1: ERLP parameters

\begin{tabular}{|l|l|l|}
\hline Parameters & & Units \\
\hline Nominal Gun Energy & 350 & $\mathrm{keV}$ \\
\hline Injector Energy & 8.35 & $\mathrm{MeV}$ \\
\hline Circulating Beam Energy & 35 & $\mathrm{MeV}$ \\
\hline RF Frequency & 1.3 & $\mathrm{GHz}$ \\
\hline Bunch Repetition Rate & 81.25 & $\mathrm{MHz}$ \\
\hline Nominal Bunch Charge & 80 & $\mathrm{pC}$ \\
\hline Maximum Train Length & 100 & $\mu \mathrm{s}$ \\
\hline Maximum Train Repetition Rate & 20 & $\mathrm{~Hz}$ \\
\hline Maximum Average Current & 13 & $\mu \mathrm{A}$ \\
\hline
\end{tabular}

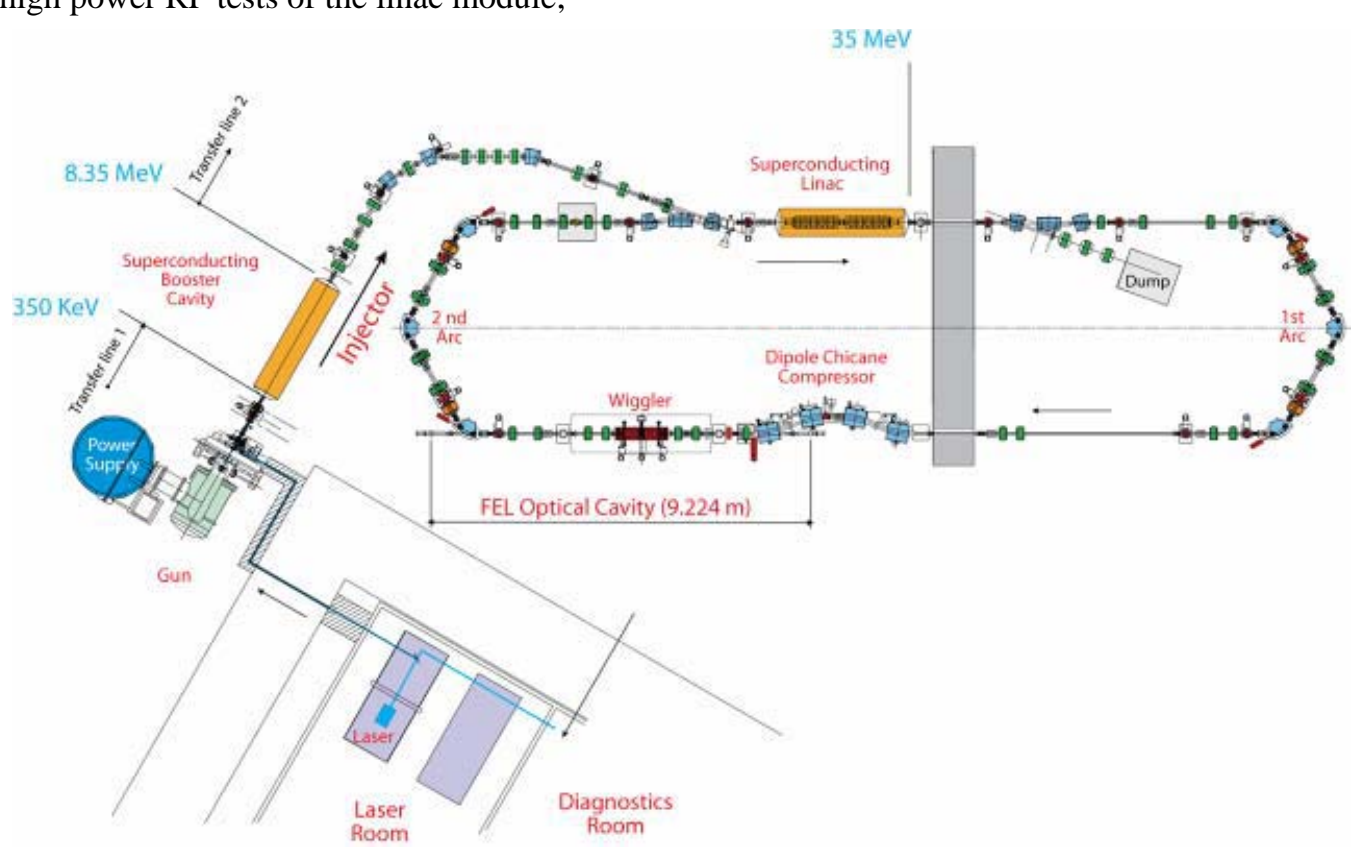

Figure 1: ERLP layout. 


\section{GUN COMMISSIONING}

The first electron beam was obtained from the gun at $250 \mathrm{keV}$ into a dedicated gun diagnostic beamline [2], in August 2006. Since then the performance of the photoinjector laser and gun combination has been developed to the point where only the measurements at high bunch charges remain. This has been undertaken in parallel with ex- and in-situ developments of the Negative Electron Affinity (NEA) cathode technology in order to reach Quantum Efficiencies (QE) of a few percent (along with a workable lifetime) required for the highest bunch charge. Problems encountered have been concentrated in two key areas. Firstly, the photocurrent monitored during cathode activation has been swamped by a significantly larger current derived from caesium ionisation. Secondly, contamination issues following cathode activation have impaired the ability of the gun to operate at the required voltage and to maintain the XHV conditions required for workable cathode lifetime.

The pulses from the photoinjector laser have been synchronised with the machine RF using a feedback control system provided by the laser manufacturer. Subpicosecond timing jitter is routinely achieved and work to reduce this to the $100 \mathrm{fs}$ level is continuing.

The results achieved so far are shown in Table 2.

\section{CONSTRUCTION PROGRESS}

As already mentioned, the gun is currently beaming into a dedicated gun diagnostic beamline. This prohibits the placement of the first linac module (the booster) in its final position. Other than this and some diagnostic devices currently being used with the gun, the electron beam transport system is fully assembled and under vacuum. Installation of photon beam transport systems required for the FEL output, Compton backscattering \& electro-optic longitudinal diagnostic laser and terahertz beamline is continuing, as is electrical and controls commissioning. This is illustrated in Fig. 2.

Table 2: ERLP commissioning results.

\begin{tabular}{|l|c|c|l|}
\hline Parameter & Specification & Measured & Units \\
\hline Beam Energy & 350 & 350 & $\mathrm{keV}$ \\
\hline Bunch Charge & 80 & 22 & $\mathrm{pC}$ \\
\hline Train Length & 100 & 100 & $\mu \mathrm{s}$ \\
\hline Train Repetition Rate & 20 & 20 & $\mathrm{~Hz}$ \\
\hline QE (in the gun) & $\sim 1$ & 1.5 & $\%$ \\
\hline QE (in the laboratory) & & 3.5 & $\%$ \\
\hline RF-laser timing jitter & $<1000$ & 650 & $\mathrm{fs}$ \\
\hline
\end{tabular}

All of the ERLP beam transport system was assembled as individual modules on single girders in an ISO Class 100 clean room, aligned and sealed with dry $\mathrm{N}_{2}$; temporary clean conditions were then established in the local work area prior to the joining of these modules. This was undertaken in order to produce exceptionally low levels of particulate contamination inside the vacuum envelope.

\section{CRYOGENIC SYSTEM}

Following several months of commissioning work and the identification and rectification of a number of problems, the system finally met its operational specification on $16^{\text {th }}$ May 2007. It is capable of simultaneously cooling both linac modules to below $2 \mathrm{~K}$ and maintaining this temperature under the dynamic load imposed by high gradient operation of the linac system.

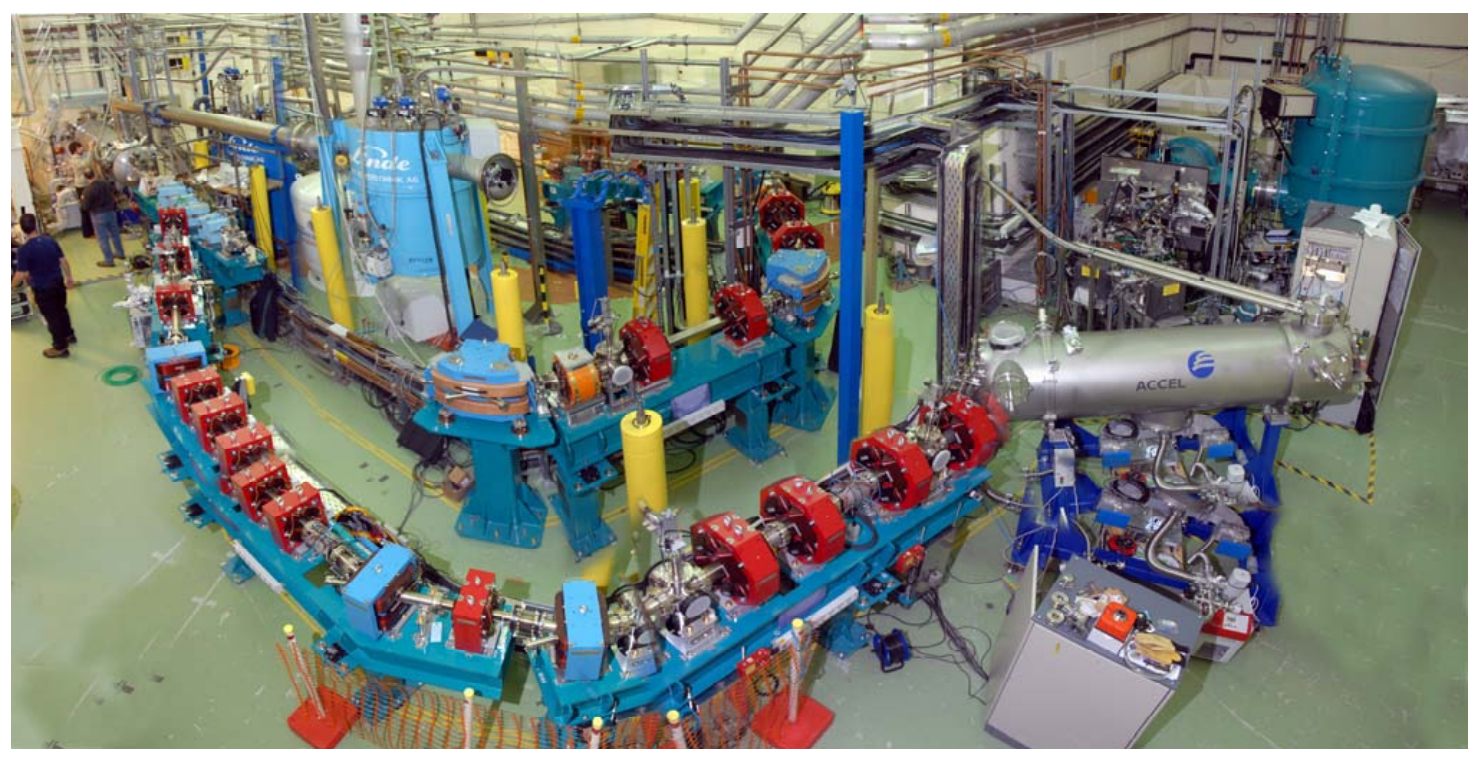

Figure 2: ERLP electron beam transport system. 


\section{HIGH-POWER RF COMMISSIONING}

At the time of writing, high-power RF tests have commenced with the main linac module, one of whose cavities has so far reached $12 \mathrm{MV} / \mathrm{m}$ (see Fig 3).

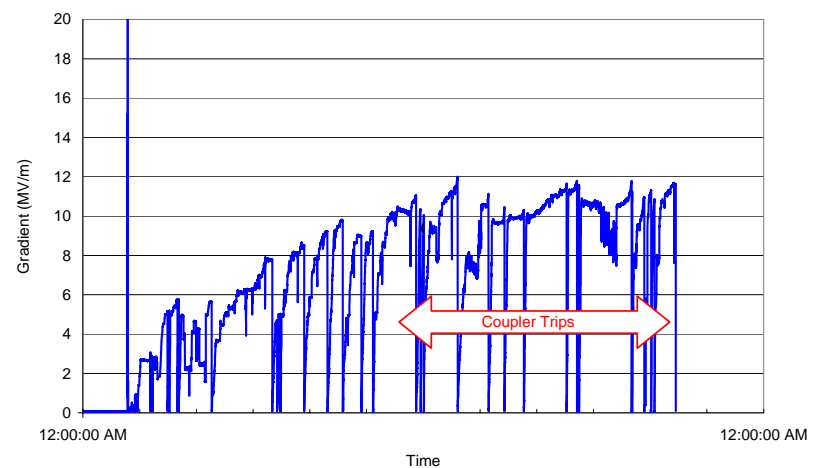

Figure 3: Main linac cavity 1 high-power RF processing.

CW conditioning of the input coupler was clearly observed, resulting in a number of vacuum and arc events at $>10 \mathrm{MV} / \mathrm{m}$. Progress has been ultimately halted however due to a mechanical failure of the tuner mechanism, which is currently being addressed. Once these have been fixed conditioning to higher gradients will continue. In addition, it has been decided to operate the booster linac in its current position, rather than waiting until the gun commissioning has finished. This will allow additional time for gun characterisation, without significantly affecting the overall programme.

\section{TERAWATT LASER}

Inverse-Compton backscattering is a means of generating, ultra-short x-ray pulses at a low-energy accelerator. An inverse COmpton BAckscattering x-ray source driven by the multi-10 TW Laser installed at Daresbury (COBALD) is being constructed [3]. Hard xrays, ranging from $15 \mathrm{keV}$ to $30 \mathrm{keV}$, depending on the backscattering geometry, will be generated by interaction between the laser pulse and the electron beam. The $\mathrm{x}$-rays created from head-on collisions contain $15 \times 10^{6}$ photons per pulse and $5 \times 10^{6}$ from side-on collisions. A highly accurate timing synchronisation scheme is employed to minimise jitter in the x-rays generated.

A commercial laser system has been purchased and installed in the laser room, alongside the photoinjector laser. This laser has now been commissioned and installation of the photon beam transport system is under way. Fig. 4 shows the two alternative (head-on and sideon) laser paths to interaction with the electron beam, just before the first dipole of the second arc. In addition, this laser will be used for the development of an electro-optic longitudinal diagnostic.

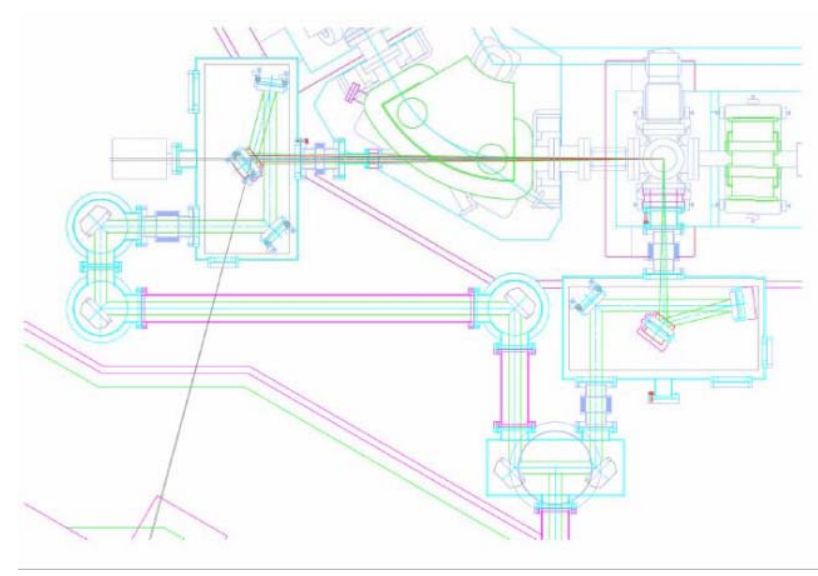

Figure 4: Laser-electron beam interaction region.

\section{CONCLUSIONS}

The prime motivation for the construction and operation of ERLP is to gain experience in designing and operating the technologies which are critical to the success of the future 4GLS machine, currently under design. These include photoinjector guns, $2 \mathrm{~K}$ cryogenics systems, superconducting linacs, transport of short-pulse, low-emittance electron beams, bunch compression, FELs and synchronisation. In the short period since the start of this project, a huge amount has been learnt, with much more still to come.

In addition to the IR radiation generated by the FEL and the x-rays produced by the Compton backscattering source, a third beamline to utilise terahertz radiation from a dipole magnet in the bunch compressor is being built. Once fully operational, ERLP will be one of the few true electron beam test facilities available in the world. It will be used for development of photoinjector guns, diagnostics, superconducting linacs, synchronisation and for benchmarking codes.

A recent addition to this list is the EMMA project [4], which is a non-scaling Fixed-Field Alternating Gradient (FFAG) technology demonstrator. This type of accelerator has many potential applications, for example for protons in radio-therapy and in neutrino factories. It will use ERLP as an injector.

\section{REFERENCES}

[1] E.A.Seddon et al, "The Status of the Daresbury Energy Recovery Prototype Project”, EPAC'06, Edinburgh, June 2007, p. 249.

[2] D.J.Holder et al, "ERLP Gun Commissioning Beamline Design", LINAC'04, Lübeck, August 2004, p. 93.

[3] G.Priebe et al, "Inverse Compton Backscattering Source Driven by the Multi-10 TW Laser Installed at Daresbury", to be published in the proceedings of SPIE Optics + Photonics, San Diego, August 2007

[4] R.Edgecock et al, "EMMA - The World's First NonScaling FFAG”, these proceedings. 